\title{
Midgap traps related to compensation processes in CdTe alloys
}

\author{
A. Castaldini, A. Cavallini, and B. Fraboni \\ INFM and Department of Physics, University of Bologna, Viale Berti Pichat 6/2, 40126 Bologna, Italy \\ P. Fernandez and J. Piqueras \\ Departamento de Fisica de Materiales, Facultad de Ciencias Fisicas, Universidad Complutense de Madrid, E-28040 Madrid, Spain
}

(Received 17 March 1997)

\begin{abstract}
We study, by cathodoluminescence and junction spectroscopy methods, the deep traps located near midgap in semiconducting and semi-insulating II-VI compounds, namely, undoped CdTe, CdTe:Cl, and $\mathrm{Cd}_{0.8} \mathrm{Zn}_{0.2} \mathrm{Te}$. In order to understand the role such deep levels play in the control of the electrical properties of the material, it appears necessary to determine their character, donor, or acceptor, in addition to their activation energy and capture cross section. Photoinduced-current transient spectroscopy and photo deep-level transient spectroscopy are used to investigate the semi-insulating (SI) samples, and a comparison of the complementary results obtained allows us to identify an acceptor trap, labeled $H$, and an electron trap, labeled $E$. Level $H$ is common to all investigated compounds, while $E$ is present only in $\mathrm{CdTe}: \mathrm{Cl}$ samples. This provides clear experimental evidence of the presence of a deep trap in $\mathrm{CdTe}: \mathrm{Cl}$, which could be a good candidate for the deep donor level needed to explain the compensation process of SI CdTe:Cl. [S0163-1829(97)08944-3]
\end{abstract}

The interest in Cd-based binary and ternary II-VI compounds arises from their promising applications as $\gamma$ - and $\mathrm{X}$-ray detectors and in electro-optic devices. The high resistivity of the material $\left(\rho \geqslant 10^{8} \Omega \mathrm{cm}\right)$ is one of the most stringent requirements, together with a high mobility-lifetime product. Semi-insulating (SI) CdTe can be obtained by growing impurity-free stoichiometric crystals or, more easily, by introducing during growth group-III or -VII donors. The dopants thus introduced act as shallow donors which compensate for the native acceptors, the cadmium vacancies $\left(V_{\mathrm{Cd}}\right)$, generated during the growth in Te-rich conditions. The resulting complex $\left(V_{\mathrm{Cd}}-\mathrm{Don}_{\mathrm{Te}}\right)$ is the so-called center $A,{ }^{1}$ which acts as a single acceptor located at $E_{V}$ $+0.15 \mathrm{eV}^{2-4}$ Its energy level is too shallow to account for the pinning of the Fermi level observed in compensated $\mathrm{Si}$ II-VI compounds, and the existence of a deep donor has been suggested to explain the compensation process. ${ }^{3,5}$ While the introduction of group- $\mathrm{V}$ impurities is known to directly introduce deep levels which play a significant role in the resulting semi-insulating behavior of the material, group-III impurities do not directly generate deep levels but only introduce shallow ones. ${ }^{6}$ In order to study the electrical activity of the deep traps, we used electrical spectroscopy methods which can be applied to SI materials, namely, PICTS (photoinduced-current transient spectroscopy) and PDLTS (photo deep-level transient spectroscopy). ${ }^{7-10}$ These techniques allow the analysis of the deep levels in a wide region of the forbidden gap, including those located near midgap, i.e., the traps which may intervene in the pinning process. Moreover, they can determine the hole or electron character of the deep levels. The characterization of the deep level optical activity has been carried out with cathodoluminescence (CL) measurements which also allow us to compare our results to those reported in the literature.

We have investigated four different sets of samples, all $p$ type: CdTe undoped $(\rho \approx 30 \Omega \mathrm{cm}), \quad \mathrm{CdTe}: \mathrm{Cl}\left(\rho>10^{7}\right.$ $\Omega \mathrm{cm})$, CdTe:Cl annealed at $T=600{ }^{\circ} \mathrm{C}$ in an $\mathrm{Ar}$ atmo- sphere for $t=5 \mathrm{~h}$, and $\mathrm{Cd}_{0.8} \mathrm{Zn}_{0.2} \mathrm{Te}\left(\rho>10^{11} \Omega \mathrm{cm}\right)$. The CdTe samples were grown with the traveling heater method, while the $\mathrm{Cd}_{0.8} \mathrm{Zn}_{0.2}$ Te ones have been grown by the highpressure Bridgman method. The undoped CdTe material is semiconducting and the $\mathrm{CdTe}: \mathrm{Cl}$ and the $\mathrm{Cd}_{0.8} \mathrm{Zn}_{0.2} \mathrm{Te}$ samples are semi-insulating. While $\mathrm{CdTe}: \mathrm{Cl}$ is made semiinsulating by the presence of $\mathrm{Cl}$ via a compensation process, $\mathrm{Cd}_{0.8} \mathrm{Zn}_{0.2} \mathrm{Te}$ is intrinsically highly resistive. Schottky diodes have been prepared by depositing a Au or In barrier on one side of the sample, while the backside Ohmic contact has been obtained by electroless Au deposition. Junction spectroscopy analyses have been carried out with a SULA Tech. system. The DLTS method has been applied to the semiconducting undoped CdTe samples, while PICTS and PDLTS have been utilized to study the semi-insulating $\mathrm{CdTe}: \mathrm{Cl}$ and $\mathrm{Cd}_{0.8} \mathrm{Zn}_{0.2} \mathrm{Te}$ samples. These last two methods are complementary, as PICTS reveals both majority and minority carrier traps without reliably allowing one to distinguish between them, and PDLTS reveals only majority carrier traps. ${ }^{8-10}$ The heating rate was $0.2 \mathrm{~K} / \mathrm{s}$, and the emission rate varied from 5 to $2 \times 10^{4} \mathrm{~s}^{-1}$. The excitation wavelength used in PICTS and PDLTS measurements were $\lambda=940$ and $880 \mathrm{~nm}$, and no major differences were observed in the resulting spectra. Cathode luminescence (CL) analyses have been performed in a Hitachi S-2500 scanning electron microscope at a temperature of $80 \mathrm{~K}$ with an accelerating voltage of $25 \mathrm{keV}$. Emission was measured with a North Coast E0-817 germanium detector.

We have focused our attention on an analysis of the carrier traps which emit at high temperature, as these are the deep levels located near midgap which mostly affect the optical and electrical properties of the investigated II-VI compounds. Figure 1 shows a CL spectrum of a CdTe:Cl sample, where two peaks, labeled $H$ and $E$, are present; the inset reports the $\mathrm{CL}$ spectrum of a $\mathrm{Cd}_{0.8} \mathrm{Zn}_{0.2} \mathrm{Te}$ sample. Figure 2 shows two PICTS spectra of a CdTe:Cl (solid line) and a $\mathrm{Cd}_{0.8} \mathrm{Zn}_{0.2} \mathrm{Te}$ (dashed line) sample, and a PDLTS spectrum, obtained in identical experimental conditions, of the $\mathrm{CdTe}: \mathrm{Cl}$ 


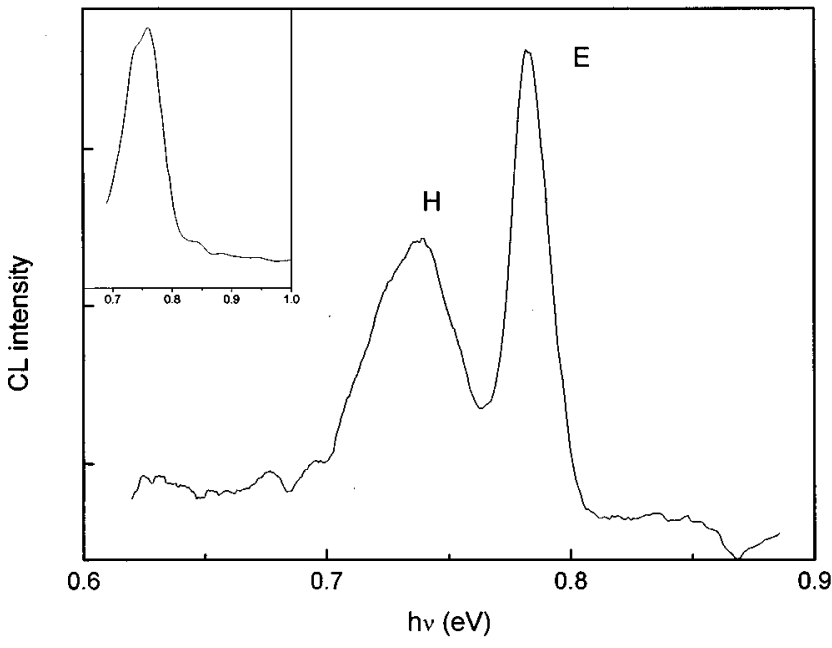

FIG. 1. Cathodoluminescence spectrum of a CdTe: $\mathrm{Cl}$ sample at $T=80 \mathrm{~K}$ and beam energy $25 \mathrm{keV}$. The inset shows a CL spectrum of $\mathrm{Cd}_{0.8} \mathrm{Zn}_{0.2} \mathrm{Te}$ under identical experimental conditions.

sample (dotted line). The same two peaks observed in CL are present, but only level $H$ is common to both materials. CL peaks may be correlated to PICTS/PDLTS since radiative transitions occur either at the energy measured in PICTS/ PDLTS or at energy values complementary to the material band gap, $E_{g}\left(E_{g}=1.54 \mathrm{eV}\right.$ for $\mathrm{CdTe}$ and $E_{g}=1.65 \mathrm{eV}$ for $\mathrm{Cd}_{0.8} \mathrm{Zn}_{0.2} \mathrm{Te}$ ). The PICTS spectrum of the CdTe:Cl samples after the annealing treatment still shows both peaks $H$ and $E$ clearly. The Arrhenius plots of the two traps are shown in Fig. 3 and the resulting activation energy and the apparent capture cross section of traps $H$ and $E$ are reported in Table I. The error associated to the activation energy has been calculated from a chi-squared fitting procedure to each data set of the Arrhenius plot and resulted to be approximately 5\% $( \pm 0.04 \mathrm{eV})$. DLTS measurements, carried out on the undoped CdTe semiconducting material, have also revealed the presence of a dominating deep trap, labeled $H_{0}$ (Table I).

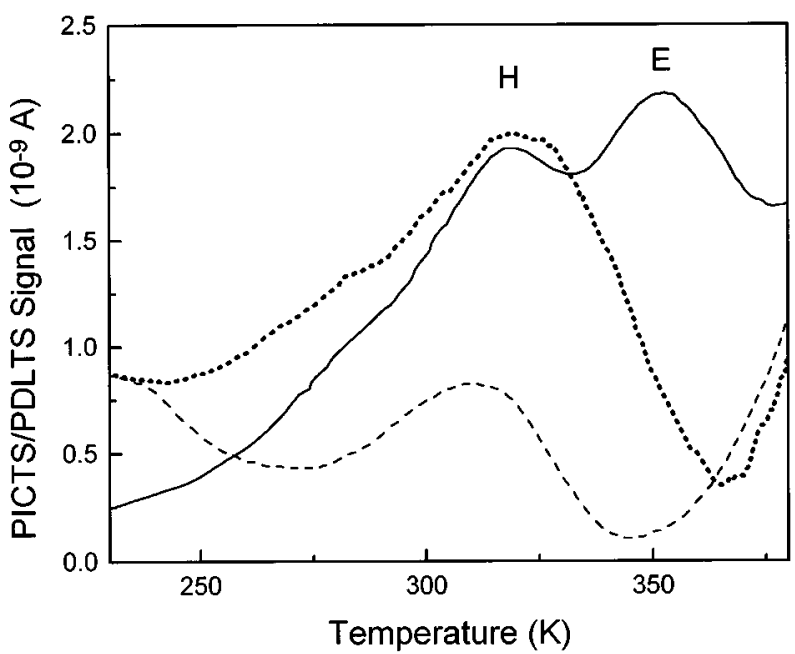

FIG. 2. PICTS (solid line) and PDLTS (dotted line) spectra of a $\mathrm{CdTe}: \mathrm{Cl}$ sample obtained with $\lambda=880 \mathrm{~nm}$ and $e_{n}=256 \mathrm{~s}^{-1}$. The PICTS spectrum of a $\mathrm{Cd}_{0.8} \mathrm{Zn}_{0.2}$ Te sample under identical experimental conditions is also reported (dashed line).

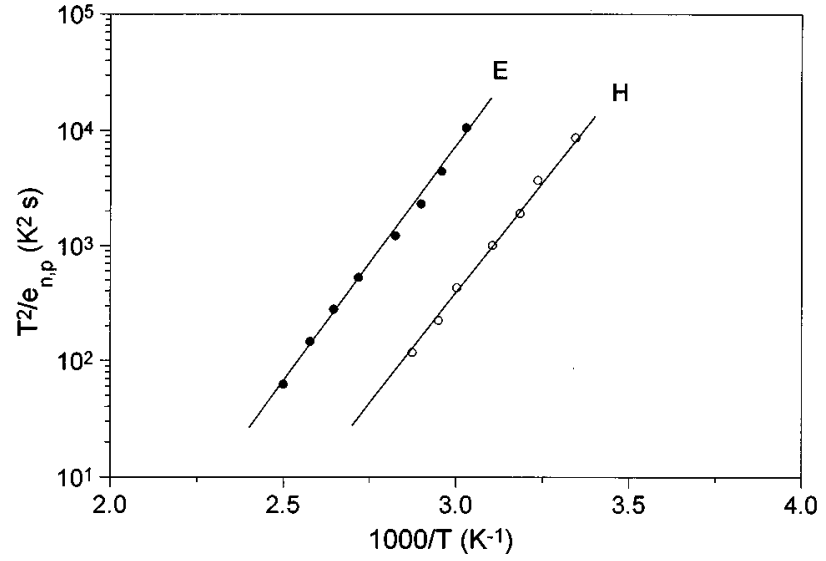

FIG. 3. Arrhenius plot of the two traps observed in the investigated materials. Experimental data are reported as solid circles; the lines represent the fit to the data sets.

Even though the investigated II-VI compounds are similar and may have identical traps, we have labeled the levels observed in DLTS and PICTS differently as the results obtained from capacitance transients (DLTS) cannot be straightforwardly compared to those derived from current transient analyses (PICTS, PDLTS): the determination of the emission rate results to be different in the two cases. ${ }^{11}$ Contrary to PICTS and PDLTS methods, for which the measurements of the trap concentration is quite difficult, ${ }^{7}$ the DLTS method allows us to determine reliably the trap concentration which we calculated for level $H_{0}$ to be $N_{H 0}=4 \times 10^{12} \mathrm{~cm}^{-3}$, taking into account the $\lambda$ effect. ${ }^{11}$ As DLTS reveals only majority carrier traps (i.e., hole traps in our case), the activation energy of level $H_{0}$ observed in undoped CdTe has to be measured from the valence band (see Table I). Conversely, it is not possible to determine the majority/minority character of the traps observed with PICTS in semiinsulating CdTe: $\mathrm{Cl}$ and $\mathrm{Cd}_{0.8} \mathrm{Zn}_{0.2}$ Te. To achieve this information it is necessary to carry out PDLTS measurements, which only reveal majority carrier traps in semi-insulating materials. The comparison of a PICTS and a PDLTS spectrum of CdTe:Cl, reported in Fig. 2, shows that the only level present in PDLTS is level $\mathrm{H}$, which, thus, results to be a hole trap. Its activation energy has to be measured from the valence band, while the activation energy of level $E$, which is an electron trap, is calculated from the conduction band (Table I). The levels found in the four different sets of samples studied are summarized in Table II.

Levels $H$ and $H_{0}$ could be attributed to the same deep trap, in particular to a level with an activation energy of approximately $0.8 \mathrm{eV}$, which has been reported in the literature for CdTe and $\mathrm{Cd}_{1-x} \mathrm{Zn}_{x}$ Te compounds grown with different methods and dopants. This deep level has been ob-

TABLE I. Summary of the activation energies and apparent capture cross sections of the levels observed in the investigated materials.

\begin{tabular}{lcc}
\hline \hline & $E_{a}(\mathrm{eV})$ & $\sigma\left(\mathrm{cm}^{2}\right)$ \\
\hline $\mathbf{H} / \mathbf{H}_{0}$ & $E_{V}+0.76$ & $6 \times 10^{-13}$ \\
$\mathbf{E}$ & $E_{C}-0.79$ & $4 \times 10^{-14}$ \\
\hline \hline
\end{tabular}


TABLE II. Summary of the levels observed in the various II-VI compounds investigated.

\begin{tabular}{lcccc}
\hline \hline & CdTe:Cl & $\begin{array}{r}\text { CdTe:Cl } \\
\text { annealed }\end{array}$ & $\begin{array}{c}\text { CdTe } \\
\text { undoped }\end{array}$ & $\mathrm{Cd}_{0.8} \mathrm{Zn}_{0.2} \mathrm{Te}$ \\
\hline $\mathbf{H} / \mathbf{H}_{0}$ & yes & yes & yes & yes \\
$\mathbf{E}$ & yes & yes & & \\
\hline \hline
\end{tabular}

served both with electrical ${ }^{12-17}$ and optical techniques, ${ }^{2,18}$ and has been widely attributed to an acceptor complex involving the native $V_{\mathrm{Cd}}{ }^{2-}$ defect and an impurity. ${ }^{14,18-20} \mathrm{~A}$ deep trap with similar characteristics has been detected in DLTS analyses of $n$-type semiconducting CdTe, ${ }^{12,15,17}$ which reveals only majority carrier traps, i.e., donor traps. It is reasonable to attribute this trap to the same deep complex observed by us, since it behaves as a recombination center. This has been deduced by measuring its thermal emission rates for electrons and holes, which results having comparable values. ${ }^{15}$ It is important to stress that, as we studied $p$-type samples, we observed the dominating majority carrier trap behavior of level $H$, but this does not imply that the level is a pure hole trap, especially when considering the midgap position of the energy level. The activation energy of level $H$ measured from the valence band is the same in CdTe and $\mathrm{Cd}_{0.8} \mathrm{Zn}_{0.2} \mathrm{Te},{ }^{16}$ and this confirms its attribution to a $\mathrm{Cd}$ sublattice defect, as the valence band (related to $\mathrm{Cd}$ defects) does not shift in energy with increasing $\mathrm{Zn}$ concentration with respect to pure CdTe. ${ }^{21}$ The acceptor character of level $H$ can be inferred from the fact that $H$ is the dominating trap in all samples, semi-insulating and semiconducting, and they all result to be $p$ type. Level $E$, on the contrary, is only observed in CdTe:Cl samples (before and after annealing), and this strongly suggests its relation to the presence of $\mathrm{Cl}$. The presence of $\mathrm{Cl}$ makes the material semi-insulating by compensating for the native acceptor defect, the $V_{\mathrm{Cd}}$. The resulting acceptor complex, the so-called center $A$, has an activation energy of $0.15 \mathrm{eV}$ which is too shallow to account for the high resistivity of the material and to justify the pinning of the Fermi level near midgap. ${ }^{2-4}$ It has been proposed in the past ${ }^{5}$ that the pinning of the Fermi level observed in $\mathrm{CdTe}: \mathrm{Cl}$ is due to its location in the gap between two major traps, an acceptor and a donor level, but such a donor level, peculiar to Cl-doped $\mathrm{CdTe}$ has, to our knowledge, been never reported in the literature. The midgap trap $E$ is present in high concentration (comparable to level $H$ ) in compensated semi-insulating $\mathrm{CdTe}: \mathrm{Cl}$, while it is absent in semicon- ducting undoped CdTe. This suggests that level $E$ could be considered the deep donor needed to explain fully the compensation process in $\mathrm{CdTe}: \mathrm{Cl}^{3,5,6,17,19} \mathrm{It}$ is worth noting that $E$ cannot be due to the thermal emission of electrons from the recombination center $H$ both because level $E$ is detected only in $\mathrm{CdTe}: \mathrm{Cl}$ samples, and because the thermal activation energy of level $H$ for electron emission has been calculated to be lower than that for hole emission, ${ }^{15}$ while, in our case, $E$ has a greater activation energy than $H$.

The role played by the levels detected in this work is different for each investigated II-VI compound. In $\mathrm{Cd}_{0.8} \mathrm{Zn}_{0.2} \mathrm{Te}$, which is intrinsically semi-insulating, level $H$ is the only deep trap located near the Fermi level at midgap and it possibly contributes to the pinning of the Fermi level. In CdTe: $\mathrm{Cl}$ there are, on the contrary, two deep levels near midgap: the acceptor level $H$, which acts as a recombination center, and the electron trap $E$, which possibly acts as a donor level and intervenes in the material compensation process. The Fermi level is probably located between these two levels and they both play an active role in its pinning near midgap. ${ }^{5}$ In undoped CdTe, which is semiconducting, level $H$ acts as a deep acceptor center and it has no pinning effect on the Fermi level which is far from midgap. We calculated the position of the Fermi level in our samples at room temperature and it resulted to be located at $E_{C}-1.32 \mathrm{eV}$ (the band gap of CdTe is $E_{g} \approx 1.54 \mathrm{eV}$ at room temperature). ${ }^{19,20}$

In conclusion, we have utilized junction spectroscopy techniques, such as DLTS, PICTS, and PDLTS, to study the deep traps located near midgap in semiconducting and semiinsulating II-VI compounds. Two deep levels have been observed, labeled $H$ and $E$. The combined application on semiconducting and semi-insulating samples of PICTS and PDLTS has allowed us to determine that $H$ is an acceptor trap, and to propose $E$ as a donor level. Level $H$ is common to all investigated materials, and has been attributed to a deep acceptor complex related to $V_{\mathrm{Cd}}{ }^{2-}$. The electron trap character of level $E$, present only in CdTe: $\mathrm{Cl}$ material, is reported here for the first time, to our knowledge. Level $E$ seems to be a good candidate for the deep donor missing in the full understanding of the compensation process in semiinsulating CdTe:Cl.

This research was partially supported by the Cooperation Programme "Azione Integrata" between Italy and Spain and by DGICYT (Project No. PB 93-1256). The authors are indebted to Japan Energy Corporation for the undoped and Cl-doped samples.
${ }^{1}$ J. W. Allen, Semicond. Sci. Technol. 10, 1049 (1995).

${ }^{2}$ D. M. Hofmann, D. Omling, H. G. Grimmeiss, B. K. Meyer, K. W. Benz, and D. Sinerius, Phys. Rev. B 45, 6247 (1992).

${ }^{3}$ W. Stadler, D. M. Hoffman, H. C. Alt, T. Muschik, B. K. Meyer, E. Weigel, G. Müller-Vogt, M. Salk, E. Rupp, and K. W. Benz, Phys. Rev. B 51, 10619 (1995).

${ }^{4}$ A. Castaldini, A. Cavallini, B. Fraboni, P. Fernandez, and J. Piqueras, Appl. Phys. Lett. 69, 3507 (1996).

${ }^{5}$ N. V. Agrinskaya and E. N. Arkadeva, Nucl. Instrum. Methods Phys. Res. A 283, 260 (1989).
${ }^{6}$ E. Rzepka, Y. Marfaing, M. Cuniot, and R. Triboulet, Mater. Sci. Eng. 16, 262 (1993).

${ }^{7}$ M. Tapiero, N. Benjelloun, J. P. Zielinger, S. El Hamd, and C. Noguet J. Appl. Phys. 64, 4006 (1988).

${ }^{8}$ O. Yoshie and M. Kamihara, Jpn. J. Appl. Phys. 22, 621 (1983).

${ }^{9}$ P. M. Mooney, J. Appl. Phys. 54, 208 (1983).

${ }^{10}$ A. Castaldini, A. Cavallini, B. Fraboni, L. Polenta, P. Fernandez, and J. Piqueras, in Electrically-Based Microstructural Characterization, edited by R. A. Gerhardt, S. R. Taylor, and E. J. Garboczi, MRS Symposia Proceedings No. 411 (Materials Re- 
search Society, Pittsburgh, 1996), p. 177.

${ }^{11} \mathrm{P}$. Blood and J. W. Orton, The Electrical Characterization of Semiconductors: Majority Carriers and Electron States (Academic, London, 1992), Chap. 9, pp. 478-492 and Chap. 7, pp. 336-380.

${ }^{12}$ C.-P. Ye and J. Chen, J. Appl. Phys. 67, 2457 (1990).

${ }^{13}$ M. Fiederle, D. Ebling, C. Eiche, D. M. Hofmann, M. Salk, W. Stadler, K. W. Benz, and B. K. Meyer, J. Cryst. Growth 138, 529 (1994).

${ }^{14}$ M. Hage-Ali and P. Siffert, Nucl. Instrum. Methods Phys. Res. A 322, 313 (1992).

${ }^{15}$ T. Takebe, J. Saraie, and H. Matsunami, J. Appl. Phys. 53, 457 (1982).
${ }^{16}$ A. Castaldini, A. Cavallini, B. Fraboni, L. Polenta, P. Fernandez, and J. Piqueras, Phys. Rev. B 54, 7622 (1996).

${ }^{17}$ Z. Huang, E. Eissler, and C. Wie, Nucl. Instrum. Methods Phys. Res. B 100, 507 (1995).

${ }^{18}$ U. Pal, P. Fernandez, J. Piqueras, N. V. Suchinski, and E. Dieguez, J. Appl. Phys. 78, 1992 (1995).

${ }^{19}$ P. Moravec, M. Hage-Ali, L. Chibani, and P. Siffert, Mater. Sci. Eng. 16, 223 (1993).

${ }^{20}$ P. Höschl, P. Moravec, J. Franc, E. Belas, and R. Grill, Nucl. Instrum. Methods Phys. Res. A 322, 371 (1992).

${ }^{21}$ D. M. Hofmann, W. Stadler, K. Oettinger, B. K. Meyer, P. Omling, M. Salk, K. W. Benz, E. Weigel, and G. Müller-Vogt, Mater. Sci. Eng. 16, 128 (1993). 